Avrupa Bilim ve Teknoloji Dergisi

Özel Sayı, S. 312-322, A ğustos 2020

(C) Telif hakkı EJOSAT'a aittir

Arastırma Makalesi
European Journal of Science and Technology

Special Issue, pp. 312-322, August 2020

Copyright (C) 2020 EJOSAT

Research Article

\title{
Bir Asenkron Motorun Mekanik Titreşim Sinyallerinin Ölçülerek Arıza Analizinin Yapılması
}

\author{
Süleyman Çeven ${ }^{1}$, Raif Bayır ${ }^{2 *}$ \\ ${ }^{1}$ Düzce Üniversitesi, Düzce Meslek Yüksekokulu, Elektronik ve Otomasyon Teknolojisi Bölümü, Düzce, Türkiye (ORCID: 0000-0002-8970-4826) \\ ${ }^{2}$ Karabük Üniversitesi, Teknoloji Fakültesi, Mekatronik Mühendisliği Bölümü, Karabük, Türkiye (ORCID: 0000-0003-3155-8771)
}

(Bu yayın 26-27 Haziran 2020 tarihinde HORA-2020 kongresinde sözlü olarak sunulmuştur.)

(DOI: 10.31590/ejosat.780063)

ATIF/REFERENCE: Çeven, S., \& Bayır, R. (2020). Bir Asenkron Motorun Mekanik Titreşim Sinyallerinin Ölçülerek Arıza Analizinin Yapılmasi. European Journal of Science and Technology, (Special Issue), 312-322.

$\ddot{O} z$

Asenkron motorlar, endüstride en yaygın kullanılan motor türüdür. Asenkron motorların verimlerinde meydana gelen bozulmalar, çalışma performanslarını ciddi şekilde etkilemektedir. Üretim sürecinin aksamaması için bu motorların bakımlarının periyodik bir şekilde yapılması ve durumlarının izlenmesi gerekmektedir. Bu çalışmada bir asenkron motorun farklı hız kademelerinde mekanik titreşimlerinin ölçülmesi için bir deney düzeneği tasarlanmış ve gerçekleştirilmiştir. Deney düzeneğinde asenkron motorun gövdesine "Z" ekseni yönünde bağlanan bir ivmeölçer yardımıyla titreşim sinyalleri elektrik sinyaline dönüştürülmüş ve bir veri alışveriş kartı üzerinden bilgisayar ortamına aktarılmıştır. Bu yöntem ile asenkron motorda gerçek zamanlı durum izleme gerçekleştirilmektedir. Motor üzerinde oluşan mekanik titreşimler ayrıca titreşim ölçümü yapabilen test cihazı ile ölçülmüş ve hafiza kartına kayıt edilmiştir. Elde edilen sensör verileri FFT dönüşümü kullanılarak analiz edilmiş ve sonuçlar yorumlanmıştır.

Anahtar Kelimeler: Asenkron motor, Gerçek zamanlı izleme, Titreşim ölçümü, FFT dönüşümü.

\section{Fault Analysis of an Induction Motor by Measuring Mechanical Vibration Signals}

\begin{abstract}
Induction motors are the most widely used motor type in the industry. The decrease in the efficiency and health of induction motors seriously affects their performance. In order for the production process not to fail, these motors should be maintained periodically and monitored for their condition. In this study, an experimental setup is designed and implemented to measure the mechanical vibrations of an induction motor at different speed levels. In the experimental setup, the vibration signals were converted into electrical signals with an accelerometer connected to the body of the iduction motor in the direction of the " $\mathrm{Z}$ " axis and transferred to the computer environment via a data acquisition card. With this method, real-time condition monitoring is provided on the induction motor. The mechanical vibrations that occur on the motor are also measured with a test device that can measure vibration and are recorded on the memory card. The sensor data obtained were analyzed using the FFT conversion and the results were interpreted.
\end{abstract}

Keywords: Induction motor, Real-time monitoring, Vibration measurement, FFT transform.

\section{Giriş}

Mekanik sistemler endüstrinin her alanında, farklı ortamlarda gerçekleştirilen üretim süreçlerinde kullanılmaktadır (Saruhan et al., 2014). Dişli mekanizmalar, rulmanlar, kayış-makara sistemleri, dönel miller mekanik sistemlerde döner hareketin ve gücün iletimi için kullanılmaktadır (X. Liu et al., 2018). Elektrik motorları elektrik enerjisini hareket enerjisine çeviren makinelerdir (Benbouzid, 2000). Elektrik motorları genel olarak stator, rotor ve gövdeden oluşmaktadır. Stator, elektrik motorunda bobin sargılarının bulunduğu ve

* Corresponding Author: Karabük Üniversitesi, Teknoloji Fakültesi, Mekatronik Mühendisliği Bölümü, Karabük, Türkiye, ORCID: 0000-0003-31558771, rbayir@karabuk.edu.tr 
motorun gövdesine bağlı olan sabit sistemdir. Rotor, elektrik motorunun döner elemanıdır ve motor gövdesine rulmanlar vasıtasıyla bağlanmaktadır. Elektrik motorları endüstride ağır yük uygulamalarında yoğun olarak kullanılması sebebiyle, bu elemanların elektriksel ve mekanik kısımlarında hasarlar ve arızalar meydana gelmektedir (Benbouzid, 2000; Cristalli et al., 2006; Tiwari et al., 2000). Elektrik motorlarında oluşacak bu arızaların önceden bilinmesi ve tespit edilmesi, üretimin daha planlı yapılmasına ve oluşabilecek daha büyük arızaların önlenmesi için gereklidir. Elektrik motorlarında mekanik arızaların tespiti, motorun çalışması sırasında motor stator akım ve gerilim sinyallerinin analiz edilmesi veya motorda meydana gelen mekanik titreşimlerin ölçülmesi ve analiz edilmesiyle mümkün olmaktadır (Bayir et al., 2004). Günümüzde mekanik titreşim analizi sayesinde elektrik motorlarında birçok mekanik arıza tespit edilebilmektedir (Areias et al., 2019; Jayakumar et al., 2017; Saruhan et al., 2014; Tiwari et al., 2000; Zhen et al., 2019). Elektrik Motoru (EM), çalışması sırasında oluşan mekanik titreşim sinyalleri, döner elemanlarda bulunan balans, rulman arızaları, mekanik kayıplar, hatalı montajlama, yapıdaki rezonans ve doğal frekanslar, EM faz sargılarındaki arızalar, EM'nun montajlı olduğu hareket aktarma mili kaynaklı arızalar, EM'nun montajlı olduğu dişli kutusundaki arızalar olmak üzere değişkenlik göstermektedir (Sudhakar et al., 2009; Tsypkin, 2011). Arızanın türü ve etkisine göre EM üzerinde mekanik titreşim sinyallerinin değişmesi sonucunda bu sinyaller çeşitli yöntemler kullanılarak değerlendirilmekte, arızanın türü ve etkisi tespit ve teşhis edilebilmektedir (Bayir et al., 2004; Sudhakar et al., 2009).

EM üzerinde kondisyon izleme, sistemin performansını korumak için, EM'nun belirgin parametrelerini sürekli izleme ve yorumlama işlemlerine verilen genel addır (Choudhary et al., 2019). Bu işlem sayesinde sistem verimi, maliyet, sistem performansı sürdürülebilir olmaktadır (D Goyal et al., 2016). Asenkron motorlar güvenilir yapıları, ucuz olmaları, sağlamlıkları ve uzun ömürlü olmaları sebebiyle endüstride en yaygın kullanılan elektrik motorlarıdır (Van Hecke et al., 2016). Asenkron motorlar demir yolları, madencilik, otomasyon, ağaç işleri, otomotiv sektörleri gibi hemen her sektörde tahrik elemanı olarak kullanılmaktadır. Literatürde asenkron motorlarda balanslı stator sargıları, kırık rotor, bozuk rotor ekseni, rotor rulmanları ve yanlış bağlantı hataları üzerinde yapılmış birçok çalışma mevcuttur (Glowacz et al., 2017; Henao et al., 2014; SaravanaKumar et al., 2009). Şekil 1'de literatürde asenkron motor arıza tipleri verilmektedir.

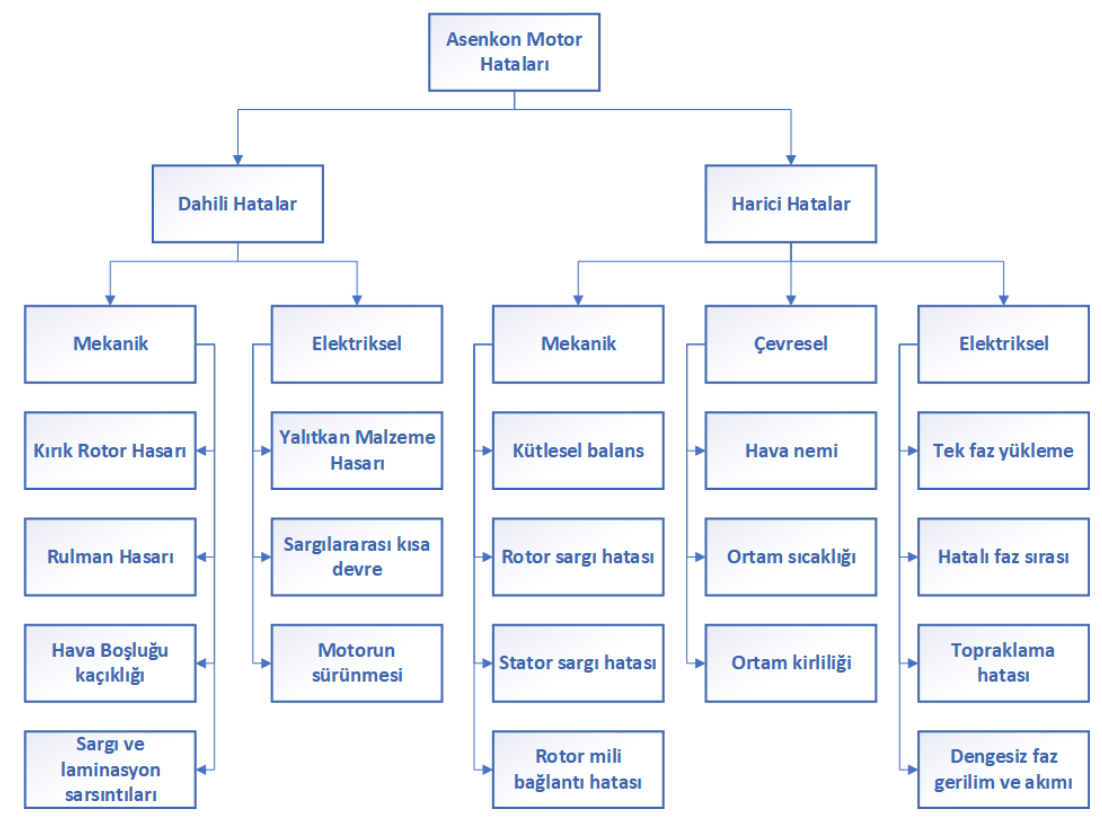

Şekil 1. Asenkron motorlarda meydana gelen hataların sınıflandırllması

Günümüz endüstrisinde kullanılan asenkron motorlarda periyodik olarak bakım işlemi gerçekleştirilmektedir. Ayrıca motorun bulunduğu ortamın çevresel ve çalışma koşulları nedeniyle asenkron motorlarda düzensiz aralıklarla arıza meydana gelebilmektedir. Dolayısıyla asenkron motorun çalışma performansının en üst düzeyde tutulabilmesi için bu motorların durumlarının gerçek zamanlı olarak izlenmesi ve parametrelerindeki anlık değişimlerin analiz edilmesi gerekmektedir. Gelişmekte olan yaklaşımlarda tesiste bulunan bakımların zamanının belirlenmesi ve periyodik bakım planının sağlanması için kondisyon izleme yöntemi ile kestirimci bir bakım algoritması ortaya koyulması amaçlanmaktadır (Medoued et al., 2009). Gerçek zamanlı izleme asenkron motorun performansını korumak için yapılmaktadır (Ilonen et al., 2005). Asenkron motorun gerçek zamanlı kondisyonlarının izlenmesi için genel olarak, akustik emisyon analizi, mekanik titreşim analizi, motor akım sinyallerinin analizi gibi yöntemler kullanılabilmektedir. Bu yöntemlerin doğrulukları yüksek, ancak kullanılan donanımların (sensör, denetleyici vs.) maliyetleri yüksektir. Gerçek zamanlı durum izleme metodu günümüzde başarıyla uygulanmaktadır. Ancak bu işlemde en etkili faktör uzman yorumlarıdır. Dolayısıyla bu metodlara ek olarak aynı amaçla çalışabilen uzman ve karar verici bir denetleme sistemine ihtiyaç duyulmaktadır (Wong et al., 2010). Kendiliğinden hata tespiti yapan sistemlerde yöntem olarak Genetik Algoritmalar (GA), Bulanık Mantık (BM), Yapay Sinir Ağı (YSA) ve Uzman Sistem (US) yaklaşımları çoğunlukla tercih edilmektedir (Hwang et al., 2009; Kochhar et al., 1997; Palmero et al., 2005; Rao et al., 2012; Sugumaran et al., 2011; Zhang et al., 2017).

Bu çalışmada titreşim sensörü ile bir asenkron motorda farklı hız değerlerinde meydana gelen mekanik titreşimler ölçülmüş, ölçülen ham sensör verileri FFT analizi ile frekans düzlemine aktarılmış ve sonuçlar literatür çalışmaları incelenerek motor üzerinde arıza olma ihtimali değerlendirilmiştir. Titreşim sensöründen gerçek zamanlı olarak alınan ham sensör verilerinin FFT ile analiz edilmesi 
ve indikatörde gösterilmesi bu motorların kullanıldığı alanlardaki bakım çalışmalarının hızlandırılmasını ve üretim veriminin artmasını sağlayacaktır. Ayrıca, asenkron motorlarda meydana gelen arızalar ve bu arızaların tespit edilmesinde tercih edilen yöntemlerden bahsedilmektedir. Makalenin birinci bölümünde, asenkron motorlarda meydana gelen arızalar, mekanik titreşimler ve titreşim analiz yöntemleri, ikinci bölümünde, kullanılan materyal ve yöntemler, üçüncü bölümünde, yapılan deneysel çalışmalar ve bulgular, dördüncü bölümünde, çalışmada elde edilen sonuçlar verilmektedir.

\subsection{Asenkron Motor Arızaları}

Asenkron motorlarda oluşan arızaların sınıflandırılması Şekil 1'de verilmektedir. Şekil 1'e göre asenkron motorlarda dahili arızalar ve harici arızalar olmak üzere arızalar ikiye ayrılmaktadır. Dahili arızalar mekanik ve elektriksel olarak ayrılırken, harici arızalar ise mekanik, çevresel ve elektriksel olarak kendi aralarında sınıflandırılmaktadır. Institution of Electrical and Electronics Engineers (IEEE) tarafından paylaşılan verilere göre dünya üzerinde asenkron motor arızalarının \%9'u rotor arızası, \%28'i stator arızası, \%41'i rulman arızası ve \%22'si diğer arızalar olarak kayıtlarda geçmektedir. Diğer yandan ASEA Brown Boveri (ABB) teşkilatı verilerine göre dünya üzerinde asenkron motor arızalarının çevresel faktör kaynaklı arızalar \%16'sını, şaft merkez hatası kaynaklı arızalar \%2'sini, rotor gövdesi kaynaklı arızalar \%5'ini, stator sargısı kaynaklı arızalar \%16'sını ve rulman kaynaklı arızalar ise \%51'ini kapsamaktadır. Electric Power Research Institute (EPRI) teşkilatı verilerine göre ise rotor arızaları toplam arızaların \%8'ini, stator arızaları \%36'sını, rulman arızaları \%42'sini oluştururken \%14'lük kısım da diğer arızalar olarak kayıtlara geçmiştir. IEEE, ABB, EPRI verilerine göre as enkron motorlarda en çok bozulan elemanın rulmanlar olduğu anlaşılmaktadır (Choudhary et al., 2019).

\subsubsection{Rulman artzalart}

Rulmanlar, en az sürtünme ve mekanik enerji kaybıyla dönel hareketin iletilmesini sağlayan mekanik parçalardır (Tiwari et al., 2000). Asenkron motorlarda en yaygın meydana gelen arızalar rulman kaynaklı arızalardır. Rulmanlarda arızalar genellikle rulmanın iç yüzük olarak tabir edilen kısmı üzerinde, dış yüzük olarak tabir edilen kısmı üzerinde ya da iki eleman arasından yuvarlanmayı sağlayan çelik bilyeler üzerinde aşınma, çentiklenme vb. gibi nedenler sonucu oluşmaktadır. Bir rulmanın yapısı ve kısımları Şekil 2'de verilmektedir (W. Y. Liu et al., 2013).

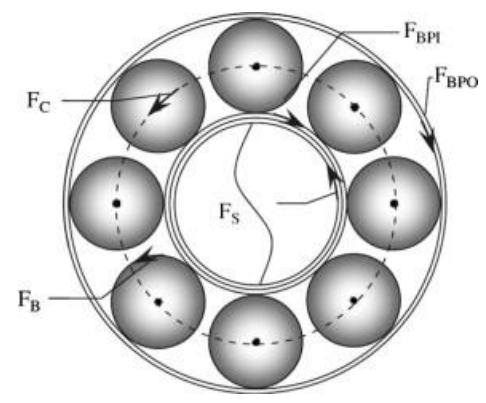

Şekil 2. Bir rulmanın yapısı

Rulman temel olarak iç halka, dış halka ve iki halka arasında bulunan küresel toplardan oluşmaktadır. Rulmanda dönme hareketi iletilirken rulman yapıları farklı hızlarda dönme hareketi gerçekleştirmektedir. Rulman kısımlarının hareket hızlarının farklı olması sonucu bu elemanların hareket frekansları da doğal olarak birbirinden farklıdır. Rulman dış halka frekansı Denklem (1)'de, iç halka frekansı Denklem (2)'de, rulman merkez frekansı Denklem (3)'te ve küresel topların frekansı ise Denklem (4)'te verilmektedir.

$$
\begin{aligned}
& F_{B P O}=\frac{\left\{N_{B}\right\}}{\{2\}} * F_{S}\left(1-\left\{D_{b} \cos \theta\right\} /\left\{D_{c}\right\}\right) \\
& F_{B P I}=\frac{\left\{N_{B}\right\}}{\{2\}} * F_{s}\left(1+\left\{D_{b} \cos \theta\right\} /\{D c\}\right) \\
& F_{c}=\frac{\{1\}}{\{2\}} * F_{s}\left(1-\left\{D_{b} \cos \theta\right\} /\{D c\}\right) \\
& F_{B}=\frac{\left\{D_{c}\right\}}{\left\{2 D_{b}\right\}} * F_{s}\left(1-\left\{D_{b}^{2} \cos ^{2} \theta\right\} /\left\{D_{c}^{2}\right\}\right)
\end{aligned}
$$

Burada, $F_{S}, F_{c}, F_{B P I}, F_{B P O}, F_{b}$ sırasıyla mil dönme frekansı, rulman merkez frekansını, iç halka frekansını, dış halka frekansını, küresel topların frekansı olarak tanımlanmaktadır. Ayrıca eşitliklerde verilen $D_{b}, N_{B}, D_{c}, \theta$ parametreler sırasıyla, küresel top çapı, küresel top sayısı, rulman merkez çapı ve küresel top temas açısı olarak tanımlanmaktadır. Sağlıklı bir rulmanın çalışması sırasında titreşim ölçümleri yapıldığında frekans düzleminde bu dört temel frekans rahatlıkla izlenebilmektedir. Ayrıca bu elemanların herhangi birinde hata olduğu anda kolaylıkla bu yolla tespit edilebilmesi mümkün olmaktadır (Zhen et al., 2019).

\subsubsection{Stator artzalart}

Asenkron motorlarda en çok karşılaşılan arıza türlerinden bir diğeri de motor stator hatalarıdır. Stator hataları genellikle, stator sargılarında, sargı laminasyon malzemelerinde, statorun gövdesini oluşturan yapıda meydana gelmektedir (Eftekhari et al., 2013). Stator üzerinde ayrıca elektriksel olarak gövdeye kısa devre arızası, açık devre arızası, fazdan faza kısa devre, sargıdan sargıya kısa devre gibi arızalar da meydana gelmektedir (Mousavi et al., 2017).

\subsubsection{Rotor arizalart}


Asenkron motorlarda rulman ve statordan kaynaklı hatalardan sonra en çok karşılaşılan arıza çeşidi rotor arızalarıdır. Rotor arızaları genellikle rotorda meydana gelen mekanik kırılma ya da rotor gövdesinde darbe sonucu oluşan çentikler sonucunda ortaya çıkmaktadır (Siddiqui et al., 2015).

\subsection{Kondisyon İzleme Teknikleri}

Endüstride bakım sürecinin en önemli adımlarından biri olan kondisyon izleme, asenkron motorun bakımının yapılması ve bakım zamanının tahmin edilmesinde fayda sağlayan bir tekniktir. Asenkron motorda kondisyonun izlenmesi ve hatanın tespit edilebilmesi için çeşitli yöntemler bulunmaktadır (D Goyal et al., 2016). Asenkron motorlarda kondisyon izleme yöntemleri, mekanik titreşim izleme, akustik ses sinyalleri izleme, termal izleme, hava aralığı torku izleme, motor akımı izleme temel olarak sınıflandırılabilmektedir (Zhang et al., 2017).

\subsubsection{Mekanik titreșim izleme}

Asenkron motorun kondisyonunun izlenmesinde en etkili yöntem titreşim izleme yöntemidir (Yamamoto et al., 2016). Asenkron motorda mekanik titreşim sinyalleri üzerinde, özellikle son yıllarda oldukça başarılı sonuçlar alınan Dalgacık dönüşüm analizi, Fast Fourier Tranform (FFT) analizi, Discrete Fourier Transform (DFT) analizi, Short Time Fourier Transform (STFT) analizi gibi yöntemler uygulanarak başarılı sonuçlar elde edilmiştir (Betta et al., 2002; de Jesus Rangel-Magdaleno et al., 2010). Asenkron motorun çalışması sirasında meydana gelen elektromanyetik vibrasyonun matematiksel ifadesi Denklem (5)'te verilmektedir. Denklem (5)'te verilen, $P(r, \omega)$ titreşim dalgasının büyüklüğ̈unü, $r$ titreşim dalgasının derecesini, $\varphi_{p}$ faz açısını, $\omega$ dalga açısal frekansını, $a$ açısal koordinatları, $t$ ise zamanı temsil etmektedir.

$$
P(a, t)=P(r, \omega) \cos \left(r a-\omega t-\varphi_{p}\right)
$$

Asenkron motorun sağlıklı bir şekilde çalıştığı durumlarda mekanik titreşim ölçülebilir. Ancak ölçüm sonucunda elde edilen dalganın genlik değeri düşük olacaktır. Asenkron motorun stator, rotor, rulman gibi sistemlerinde meydana gelen bir arızanın mekanik titreşim dalgalarında genlik değerini arttırması ve frekans düzlemi incelendiğinde farklı frekanslarda harmoniklerin oluşması, ilaveten arıza sinyalleri belirgin bir şekilde ortaya çıkması beklenmektedir (Raghavendra et al., 2014). Mekanik titreşim sinyallerinin analiz edilmesinde kullanılan en etkili metod, sinyalin frekans düzleminde analiz edilmesi ile sağlanmaktadır (Datta et al., 2016). Zaman düzleminde ölçülen bir mekanik titreşim sinyalinin frekans düzlemine aktarılması Denklem (6) ile sağlanmaktadır.

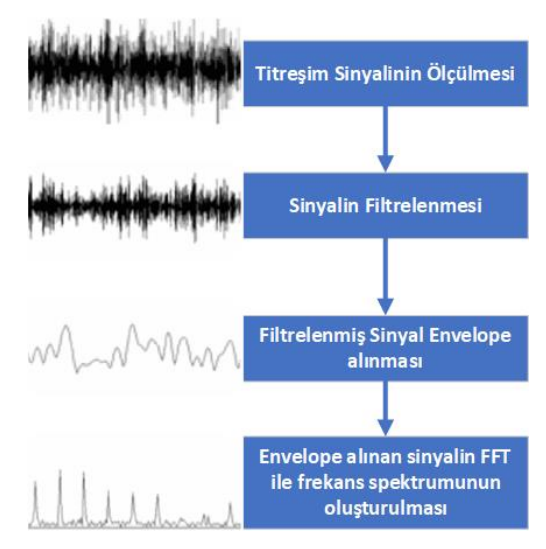

Şekil 3. Envelope analizi akış şeması

Titreşim analizinin yapılmasında kullanılan bir diğer önemli metod ise Envelope analizidir. Envelope analizi temel olarak üç adımdan oluşmaktadır. Bunlar, sinyal filtreleme, Hilbert dönüşümü ile filtrelenmiş sinyalin Envelope işleminin yapılması, Envelope alınan sinyalin FFT ile frekans spektrumunun ortaya çıkartılması olarak sınıflandırılabilmektedir. Denklem (7)'de Hilbert dönüşümü verilmektedir. Şekil 3'te analizin akışşeması verilmektedir.

$$
\begin{aligned}
& X(f)=\int_{-\infty}^{+\infty} x(t) e^{-i 2 \pi f t} d t \\
& \hat{x}(t)=\frac{1}{\pi} \int_{-\infty}^{+\infty} \frac{x(\tau)}{t-\tau} d \tau \\
& \operatorname{STFT}_{x}^{\omega}(\tau, f)=\int x(t) \omega(t-\tau) e^{-i 2 \pi f t} d t
\end{aligned}
$$

Titreşimin analizinin yapılmasında yaygın olarak kullanılan bir diğer yöntem de STFT yöntemidir. STFT yöntemi ile bir titreşim sinyali eşit periyotlarda bölünerek, oluşturulan pencerelerde ayrı ayrı FFT analizinin alınması işlemine dayanmaktadır. Bu işlem sonunda sinyal zaman-frekans düzeminde gösterilebilecektir. Bir çok çalışmada yaygın olarak kullanılan bir yöntemdir (Mohammed et al., 2016; Pang et al., n.d.; Prudhom et al., 2017). STFT'ye ait matematiksel ifade Denklem (8)'de, çalı̧̧ma blok diyagramı Şekil 4'te verilmektedir. 


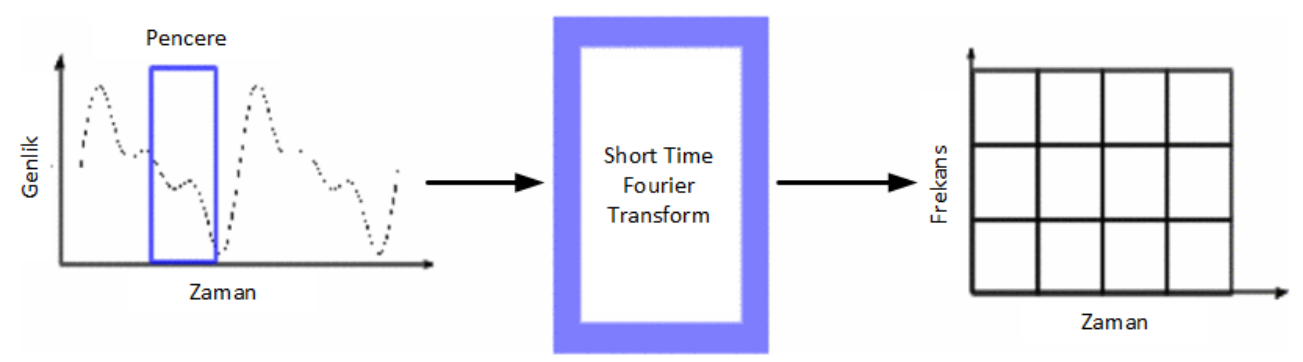

Şekil 4. STFT çalışma blok diyagramı

Titreşim sinyalleri frekans düzleminde analiz edilebildiği gibi zaman düzleminde de analiz edilebilmektedir. Titreşim sinyallerinin zaman düzleminde analizinin gerçekleştirilebilmesi için çeşitli istatistiksel yöntemlere ihtiyaç duyulmaktadır (Igba et al., 2016). Bu yöntemler sayesinde titreşim sinyalleri zamana göre analiz edilebilecek ve gerçek zamanlı olarak izlenebilecektir. Titreşim sinyalleri literatürde genel olarak, Root Mean Square (RMS), Crest Factor (CF), Standart Deviation (SD), Kurtosis metodu, Sinyal Shape Factor (SSF), Energy Ratio (ER), Energy Operator (EO), Zero Order Figure of Merit (FMo), Fourth Order Figure of Merit (FM4), M6A metodu, M8A metodu, NA4 metodu, NA4* metodu, NB4 metodu, Delta RMS metodu, Sideband Level Factor, Sideband Index, CAL4, Clearance Factor, Impulse Indicator, Correlated Kurtosis, Fourth Order Normalized Power (NP4) metodları kullanılabilmektedir (D Goyal et al., 2016). Literatürde yapılan çalışmalar incelendiğinde asenkron motorlarda titreşim sinyalleri üzerinde en çok kullanılan istatistiksel metodların, RMS, CF, Kurtosis metodları olduğu görülmektedir. RMS yöntemi bir sinyalin genliğini ve kuadratik ortalamasının alınmasında en yaygın kullanılan yöntemdir. RMS, sinyalden alınan örneklerin karelerinin ortalamasının karekökü alınarak hesaplanmaktadır. Bir sinyalin RMS değeri Denklem 9'dan faydalanarak bulunmaktadır.

$$
\begin{aligned}
& \mathcal{Y}_{r m s}=\sqrt{\frac{1}{N}\left[\sum_{k=1}^{N}\left(\mathcal{Y}_{k}\right)^{2}\right]} \\
& C F=\frac{y_{p k}-p k}{y_{r m s}} \\
& \text { Kurtosis }=\frac{N \sum_{k=1}^{N}\left(y_{k}-\bar{y}\right)^{4}}{\left[\sum_{k=1}^{N}\left(y_{k}-\bar{y}\right)^{2}\right]^{2}}
\end{aligned}
$$

Burada, $\mathcal{Y}$ ham zaman sinyalini, $N$ örnek sayısını, $k$ ise örnekleme indeksini temsil etmektedir. CF yöntemi ile asenkron motorlarda arızanın erken teşhis edilmesinde yaygın olarak kullanılan bir metoddur (Shnibha et al., 2012). CF hesaplanırken sinyalin RMS değeri kullanılmaktadır. CF matematiksel ifadesi Denklem (10)'da verilmektedir. Denklem (10)'da $\mathcal{Y}_{p k}-p k$ sinyalin tepeden tepeye farkını vermektedir. Literatürde dönen makinalarda arıza analizi yapılırken bu faktörün $C F=2-6$ arası değer üretmesi beklenmektedir (D Goyal et al., 2016). Eğer $C F=6$ değerinden büyükse asenkron motorda bir arıza oluştuğu düşünülebilir. Titreşim sinyallerinden kondisyon izleme yöntemlerinden bir diğeri de Kurtosis analizidir. Kurtosis analizi prensip olarak değişken bir sinyalin dördüncü dereceden standartlaşmış momentini vermektedir. Sinyal üzerinde hesaplanan Kurtosis değeri "3"'ün üzerinde hesaplanırsa asenkron motorda bir arıza oluştuğu ön görülebilmektedir (Sait et al., 2011). Kurtosis metodunun matematiksel ifadesi Denklem (11)'de verilmektedir.

\subsection{Asenkron Motorlarda Kondisyon İzlemede Kullanılan Zeki Yöntemler}

Asenkron motorlarda arızanın erken bir süreçte tespit edilmesinde genel olarak literatürde, uzman ve zeki sistemler üzerinde yapılmış çalışmalar bulunmaktadır. Zeki sistemler kullanılarak asenkron motorda oluşan titreşimlerin analizi yapılmakta ve asenkron motorlarda hata tespiti mümkün kılınmaktadır. Asenkron motorlarda kullanılan en etkili zeki sistemler, Bulanık Mantık (BM), Yapay Sinir Ağı (YSA), Support Vector Machine (SVM) ve Adaptive Neuro Fuzzy Inference System (ANFIS) yapılarıdır (Deepam Goyal et al., 2017).

\subsubsection{Yapay sinir ăg}

YSA yapıları asenkron motorda meydana gelen arızanın tespit edilmesinde kullanılan en yaygın zeki yöntemlerden bir tanesidir. Dezavantajı ise her bir motorda ayrı ayrı modellenmesi gerekmesidir. Aynı zamanda YSA nın başarılı bir şekilde çalışması için eğitilmesi gerekmektedir. Bu durumda herbir asenkron motorun yapısı ve modeli değişkenlik göstermesi hesaba katılırsa daha özel bir yöntem olarak uygulanması düşünülebilir (Bin et al., 2012; Leung et al., 2003; Saravanan et al., 2010; Unal et al., 2014).

\subsubsection{Bulanık mantık}

Asenkron motorlarda arıza tespit yöntemlerinden biri de bulanık mantık yöntemidir. BM sayesinde uzman denetiminde kural tabanı oluşturulabilmekte ve bu kural tabanı ile ölçülen titreşim sinyalleri çeşitli yöntemlerle yorumlanabilir hale getirildikten sonra değerlendirilebilmektedir. Asenkron motorlarda hata tespiti üzerinde literatürde BM ile yapılmış bir çok çalışmaya ulaşılabilmektedir (Nejjari et al., 1999; Wang et al., 2006).

\subsubsection{Adaptive Neuro Fuzzy Inference System (ANFIS)}

Temelde, bir BM yapısına YSA yapısı entegre edilerek ortaya çıkan yaklaşım çeşididir. Çeşitli çalışmalarda uygulanmış ve asenkron motorlarda arıza tespitinde kullanılabildiği düşünülmektedir (Yang et al., 2009) Asenkron motorlarda stator sargı hatası, rulman yataklarında meydana gelen aşınmalar gibi hataları başarıyla tespit ettiği literatürden anlaşılmaktadır (M S Ballal et al., 2006; Makarand S Ballal et al., 2007). 


\subsubsection{Support Vector Machines (SVM)}

SVM hem sınıflandırma hem de regresyon uygulamalarında başarıyla kullanılan bir makine öğrenme algoritmasıdır. Ayrıca kondisyon izleme uygulamalarında başarıyla uygulanabilir bir yaklaşımdır. SVM ile yapılan çalışmalar ve YSA ile yapılan çalışmalar değerlendirildiğinde SVM yöntemidinin YSA’ya kıyasla daha kararlı olduğu görülmektedir (Widodo et al., 2007).

\section{Materyal ve Metod}

$\mathrm{Bu}$ çalışmada, bir asenkron motorun çalışması sırasında titreşiminin ölçülmesi için bir deney düzeneği tasarlanmış ve gerçekleştirilmiştir. Asenkron motor çalışması sırasında titreşimleri ölçülmüş, ölçülen titreşim sinyalleri gerçek zamanlı olarak Matlab/Simulink ortamında izlenmiştir. Titreşim sinyalleri, farklı rotor hızları altında çalışan asenkron motordan ayrı ayrı ölçülmüş ve kaydedilmiştir. Asenkron motor çalışması sırasında, ayrıca vibrasyon ölçüm uygulamaları için üretilmiş endüstriyel bir ölçüm cihazı kullanılarak titreşimler ölçülmüss ve analiz edilmiştir. Titreşim analizleri, sinyallere FFT uygulanarak analiz edilmiştir. Deney düzeneği çalışma blok diyagramı Şekil 5 'te verilmektedir. Çalışma için hazırlanan deney düzeneği Şekil 6'da verilmektedir. Çalışmada kullanılan asenkron motorun özellikleri Tablo 1'de verilmektedir. Çalışmada kullanılan asenkron motor ve titreşim sensörü Şekil 7(a)'da verilmektedir.

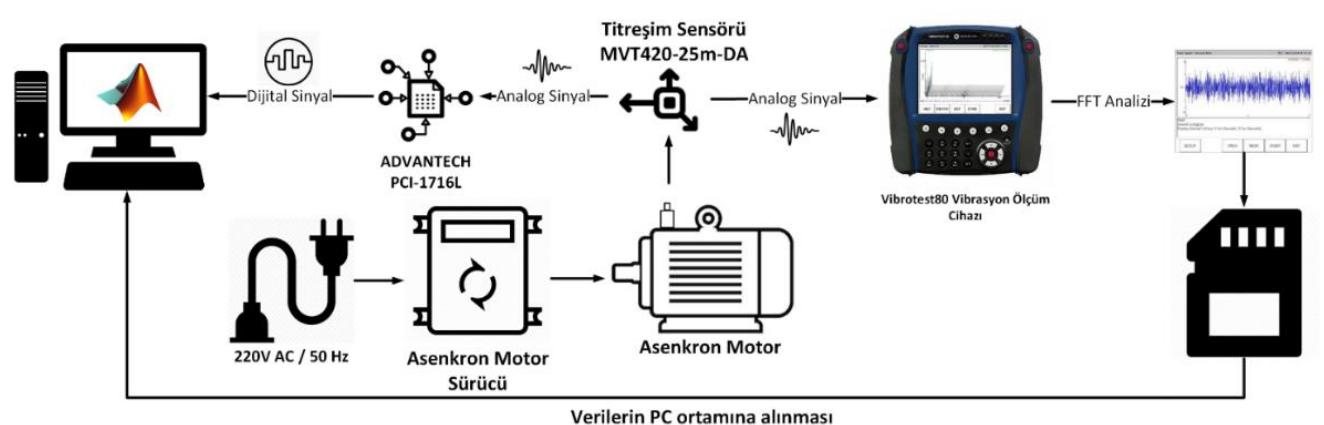

Şekil 5. Deney düzeneği çalışma blok diyagramı

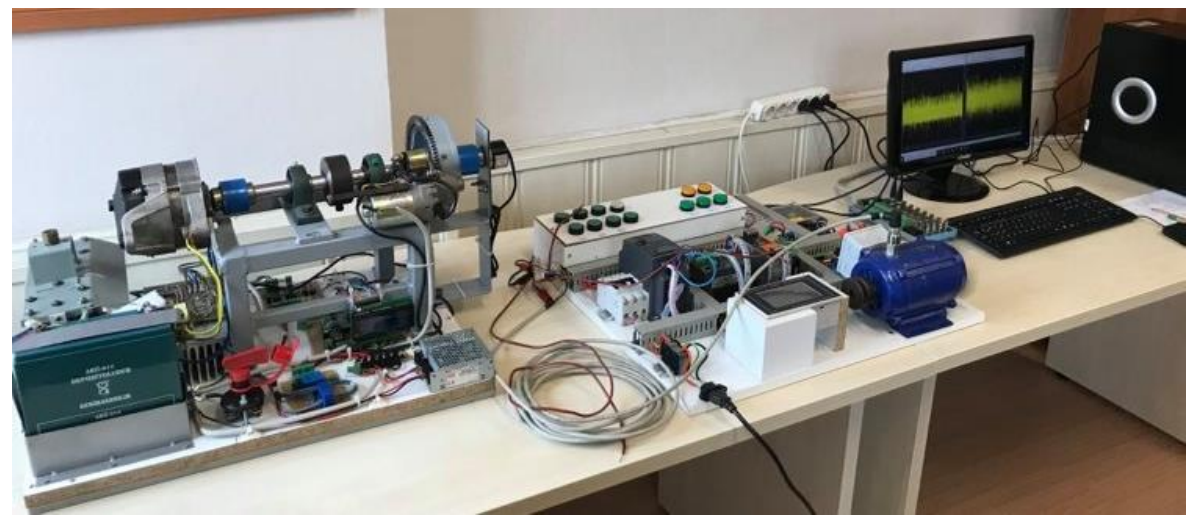

Şekil 6. Hazırlanan deney düzeneği

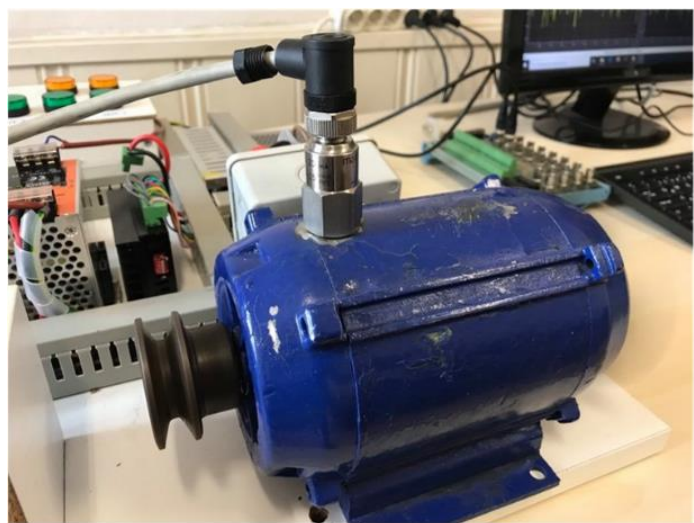

(a)

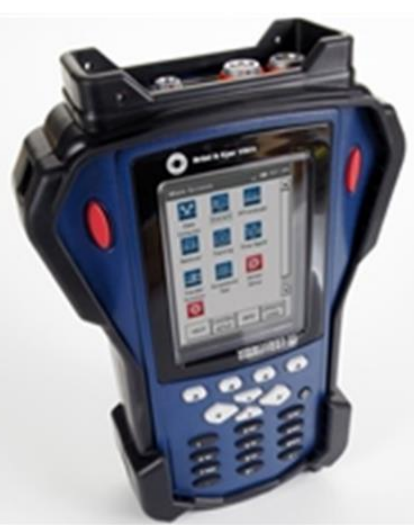

(b)

Şekil 7. Çalışmada kullanılan asenkron motor ve ölçüm cihazı, (a)asenkron motor, (b) Vibrotest80 ölçüm cihazı

Asenkron motor üzerine montajı yapılan Marmatek marka MVT-420-25mA-DA model titreşim sensörü sayesinde motorun titreşim sinyalleri ölçülmekte ve Advantech PCI1716L veri alışveriş kartı kullanılarak bilgisayara aktarılmaktadır. Bilgisayar ortamına alınan titreşim sinyalleri Matlab/Simulink ortamında gerçek zamanlı olarak izlenmektedir. Gerçek zamanlı olarak izlenen titreşim sinyalleri üzerinde FFT dönüşümü uygulanmaktadır. Ayrıca çalışmada Brüel and Kjær Vibro marka Vibrotest80 ölçüm cihazı kullanılarak titreşim 
ölçümü yapılmış ve cihaz üzerinde FFT analizi uygulanmıştır. Cihaz üzerinde yapılan ölçümler SD kart üzerine kayıt edilmiş ve bilgisayar ortamına alınmıştır. Ölçüm cihazında yapılan analizler deneysel çalışma sürecinde gerçek zamanlı olarak yapılan analizlerle karşılaştırılmış ve yöntemin doğruluğu karşıllaştırılmıştır.

Table 1. Asenkron Motor Özellikleri

\begin{tabular}{l|c}
\hline Parametre & Özellik \\
\hline Motor tipi & 3 fazlı asenkron motor \\
\hline$\Delta$-Y/220-380 & $6 / 3.5 \mathrm{~A}$ \\
\hline Nominal Güç & $1 \mathrm{~kW}$ \\
\hline Devir/Dk & $1800-50 \mathrm{~Hz}$ \\
\hline $\operatorname{Cos} \Phi$ & 0.88 \\
\hline
\end{tabular}

Çalışmada kullanılan ölçüm cihazının görseli Şekil 7(b)'de verilmektedir. Çalışmada kullanılan titreşim sensörü 14 kHz ölçüm frekansına, hız ve ivme çıkışına, IP67 koruma sınıfına sahip 4-20 mA çıkış veren endüstriyel bir sensördür. Çalışmada kullanılan veri alışveriş kartı Advantech PCI-1716L donanımı 16 kanal analog girişe, 16 bit çözünürlüğe, 250 kS/s veri okuma hızına, 1024 örnekleme sayısına sahip profesyonel bir sistemdir. Titreşim sensörü Şekil 8(b)'de verilmektedir.

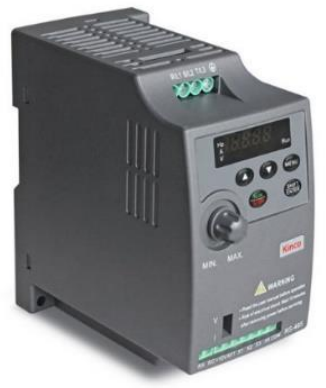

(a)

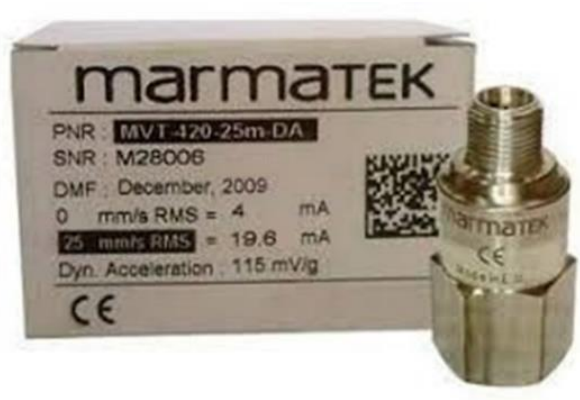

(b)

Şekil 8. Çalışmada kullanılan inverter ve titreşim sensörü, (a) CV20 model üç fazlı invertör, (b) MVT-420-25mA-DA titreşim sensörü

Çalışmada asenkron motorun kademeli olarak hızının kontrol edilmesinde KINCO marka CV20 model üç fazlı invertör kullanılmıştır. İnvertör Şekil 8(a)'da verilmektedir Gerçek zamanlı olarak ölçülen titreşim sinyallerinin Matlab mscript derleyicisi kullanılarak FFT dönüşümleri yapılmıştır. Çalışmada titreşim analizinde literatürde en çok kullanılan metod olduğu için FFT dönüşümü tercih edilmiştir. FFT dönüşümü pseudocode formatında Algoritma 1'de verilmektedir.

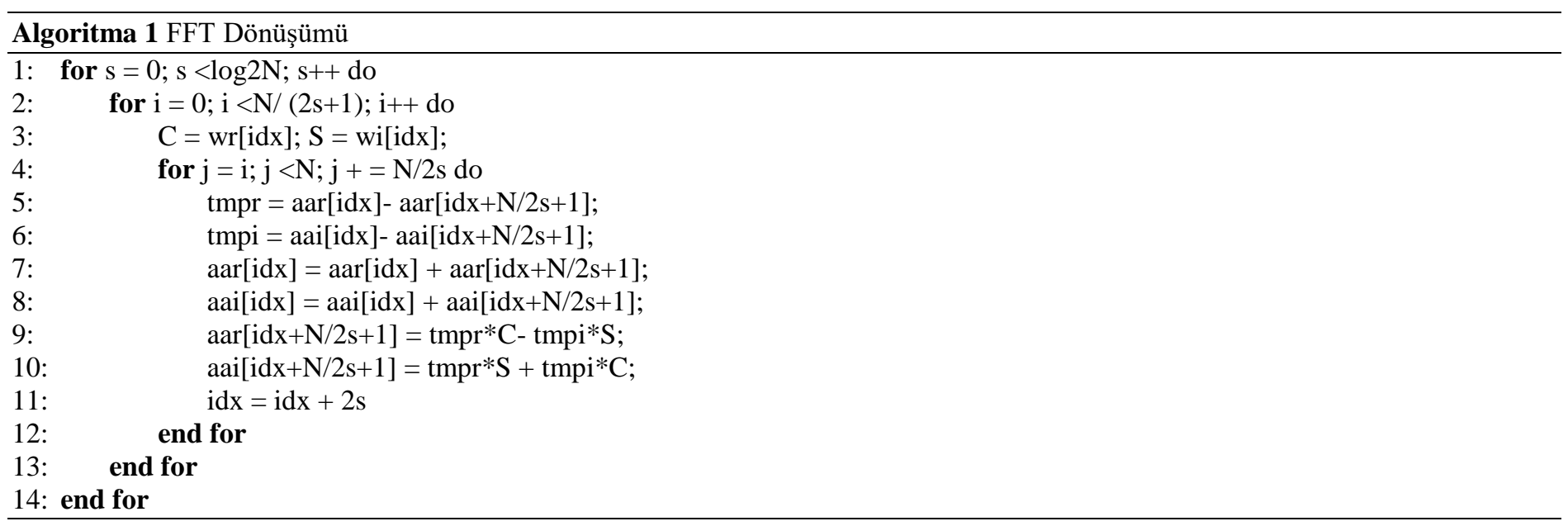

\section{Deneysel Çalışmalar}

Asenkron motor deney düzeneğinde bulunan inverter yardımıyla kademeli olarak 360 rpm, 720 rpm, 1080 rpm, 1440 rpm ve 1800 rpm rotor hızlarında çalıştırılmış ve titreşim ölçümleri yapılmıştır. Ölçümleri yapılan titreşim sinyallere Matlab ortamında FFT dönüşümü uygulandığında dalga frekanslarında meydana gelen artma gözlemlenmiştir. Şekil 9'da farklı rotor hızlarında meydana gelen titreşimlerin FFT analiz sonuçları verilmektedir. Asenkron motorun $360 \mathrm{rpm}$ hızda yapılan deneylerde elde edilen sonuçlar Şekil 10'da, 720 rpm hızda elde edilen sonuçlar Şekil 11(a)'da, 1080 rpm hızda elde edilen sonuçlar Şekil 11(b)'de, $1440 \mathrm{rpm}$ hızda elde edilen sonuçlar Şekil 12(a)'da, 1800 rpm hızda elde edilen sonuçlar Şekil 12(b)'de verilmektedir. 


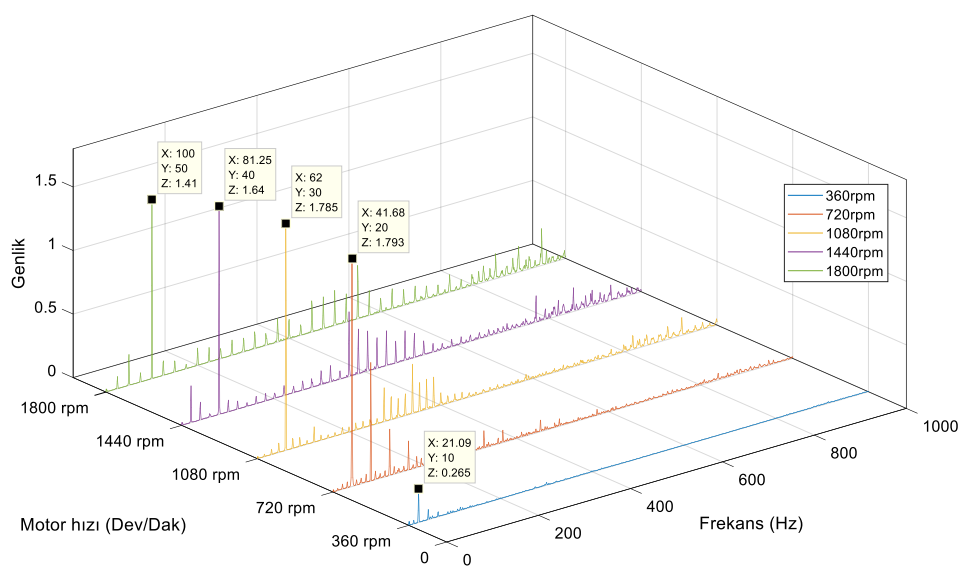

Şekil 9. Asenkron motorun farklı rotor hızlarında titreşiminin FFT analizi.
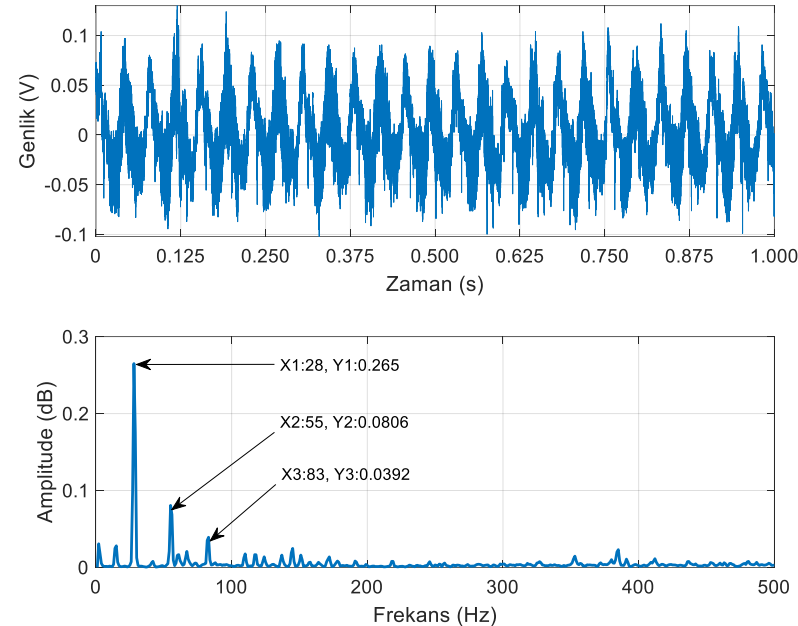

Şekil 10. 360 rpm hızda yapılan titreşim analizi

Şekil 10 incelendiğinde (X1, Y1) spektruma sahip sinyali (X2, Y2) ve (X3, Y3) spektruma sahip sinyallerin izlediği görülmektedir. Burada yaklaşık olarak X3 $=3 \mathrm{X} 1, \mathrm{X} 2=2 \mathrm{X} 1$ olduğu anlaşılmaktadır. $\mathrm{Y} 1=0.265, \mathrm{Y} 2=0.0806, \mathrm{Y} 3=0.0392$ olduğu düşünülürse buradan $(\mathrm{X} 2$, Y2) ve (X3, Y3) sinyallerinin (X1, Y1) sinyalinin harmoniği olduğu ortaya çıkmaktadır. Literatür incelendiğinde asenkron motor çalışması sonucu bu tip bir FFT sonucu çıkması asenkron motorun bu hız seviyesinde rezonansa girdiğini göstermektedir (Talhaoui et al., 2014).
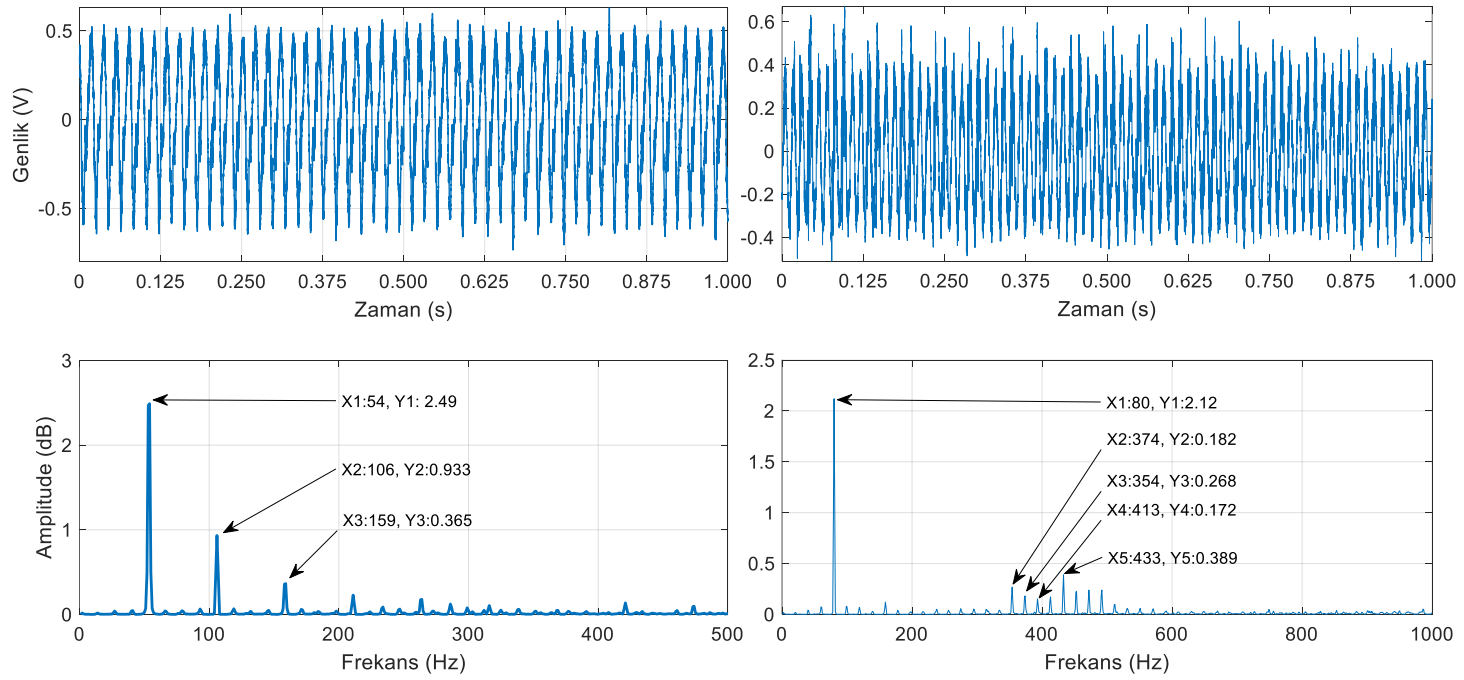

(b)

Şekil 11. (a)720 rpm hızda, (b) 1080 rpm hızda yapılan titreșim analizi 
Şekil 11(a) incelendiğinde (X1, Y1) spektruma sahip sinyali (X2, Y2) ve (X3, Y3) spektruma sahip sinyallerin izlediği görülmektedir. Burada yaklaşık olarak X3 $=3 \mathrm{X} 1, \mathrm{X} 2=2 \mathrm{X} 1$ olduğu anlaşılmaktadır. Aynı şekilde bu analiz sonucunda da $(\mathrm{X} 2$, Y2) ve (X3, Y3) sinyallerinin (X1, Y1) sinyalinin harmoniği olduğu ortaya çıkmaktadır. Dolayısıyla iki farklı hız kademesinde alınan sonuçlar değerlendirildiğinde motorun bu hız seviyesinde de rezonansa girdiğini göstermektedir. Şekil 11(b) incelendiğinde (X1, Y1) spektruma sahip sinyali (X2, Y2), (X3, Y3), (X4, Y4), (X5, Y5) spektruma sahip sinyaller izlemektedir. Burada $360 \mathrm{rpm}$ ve $720 \mathrm{rpm}$ de yapılan deneylerin aksine harmonik titreşim sinyallerinin (X1, Y1) sinyal frekansından oldukça büyük bir değere sahip olduğu görülmektedir. Ancak Y1=2.12 olması sinyal kuvvetinin şiddetli olduğunu göstermektedir. Sinyal kuvvetinde elde edilen bu değer sonucunda motorun arızalı olduğu ve literatüre dayanarak motorda rotor balansında arıza olduğu söylenebilir.
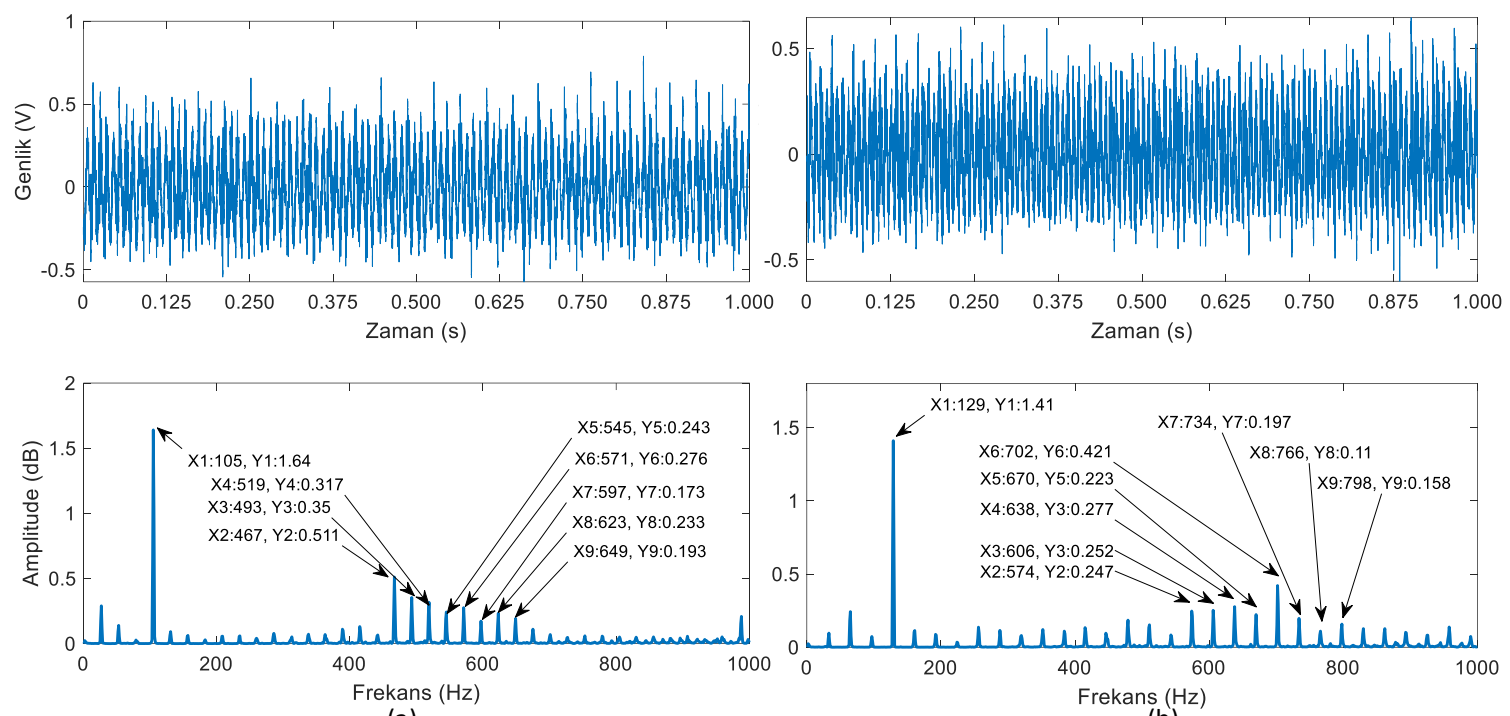

(a)

(b)

Şekil 12. (a) $1440 \mathrm{rpm}$ hızda, (b) $1800 \mathrm{rpm}$ hızda yapılan titreşim analizi

Şekil 12(a) incelendiğinde (X1, Y1) spektruma sahip sinyali (X2, Y2), (X3, Y3), (X4, Y4), (X5, Y5), (X6, Y6), (X7, Y7), (X8, Y8), (X9, Y9) spektruma sahip sinyaller izlemektedir. Burada $1080 \mathrm{rpm}$ de yapılan deneylerde alınan sonuçlara yakın bir sonuç meydana geldiği görülmektedir. Y1=1.64 olmasına rağmen harmonik sinyallerin kuvvetleri göz önüne alındığında yine titreşimin güçlü olduğu anlaşılmaktadır. Şekil 12(b) incelendiğinde (X1, Y1) spektruma sahip sinyali (X2, Y2), (X3, Y3), (X4, Y4), (X5, Y5), (X6, Y6), (X7, Y7), (X8, Y8), (X9, Y9) spektruma sahip sinyaller izlemektedir. Elde edilen sonuçlar $1440 \mathrm{rpm}$ de yapılan deneylere benzerlik göstermektedir. Yapılan deneysel çalışmalarda elde edilen bulgular yorumlandığında asenkron motorda arıza olduğu net bir şekilde ortaya çıkmaktadır.

\section{Sonuçlar}

Asenkron motorlar endüstrinin hemen her yerinde kullanılan en yaygın motor tipidir. Üretim süreçlerinin en etkili kısımlarında görev yapan bu motorların bakımlarının yapılması performanslarını ve verimlerini korumalarını sağlamaktadır. Asenkron motorlarda meydana gelebilecek arızaların önceden kestiriminin yapılması, üretimin aksamaması ve bakım maliyetlerinin minimum seviyede tutulması açısından oldukça önemlidir. Bu çalışmada bir asenkron motorun farklı rotor hızlarında titreşimleri gerçek zamanlı olarak izlenmiş ve titreşim analizleri bilgisayar ortamında gerçekleştirilmiştir. Çalışma sonucunda asenkron motorlarda titreşim analizinin, arıza tespitinde önemli bir yöntem olduğu görülmüştür. Literatürdeki çalışmalar göz önüne alındığında zeki bir sistem ile uzman yorumuna ihtiyaç duymaksızın titreşimlerin analiz edilebileceği ve zeki bir karar destek sistemi ile arızaların tahmin edilebileceği öngörülmektedir. Titreşim analizinde ham sensör sinyallerinin anlaşılmasının zorluğu neticesinde sinyalin istatistik yöntemleri ya da frekans spektrum analizleri olmadan analiz edilemeyeceği kanısına varılmıştır. Gelecek çalışmalarda makine öğrenmesi metodları kullanılarak modern bir arıza tespit ve teşhis sistemi geliştirilecek ve sistemin başarımının arttırılması üzerinde çalışmalar yapılması amaçlanmaktadır.

\section{Teșekkür}

Bu çalışma, Karabük Üniversitesi Lisansüstü Eğitim Enstitüsü doktora dersleri kapsamında üretilmiştir. Çalışmada ölçüm sonuçlarını doğrulamak amacıyla titreşim ölçüm cihazının kullanımında desteklerini esirgemeyen Düzce Üniversitesi öğretim üyesi Sayın Prof. Dr. Suat Sarıdemir ve Sayın Öğr. Gör. Zafer Cingiz’e teşekkürlerimizi sunarız.

\section{References}

Areias, I. A. dos S., da Silva, L. E., Bonaldi, E. L., de Lacerda de Oliveira, L. E., Lambert-Torres, G., \& Bernardes, V. A. (2019). Evaluation of Current Signature in Bearing Defects by Envelope Analysis of the Vibration in Induction Motors. Energies, 12(21). doi: $10.3390 /$ en 12214029

Ballal, M S, Khan, Z. J., Suryawanshi, H. M., \& Sonolikar, R. L. (2006). Induction motor: fuzzy system for the detection of winding 
insulation condition and bearing wear. Electric Power Components and Systems, 34(2), 159-171.

Ballal, Makarand S, Khan, Z. J., Suryawanshi, H. M., \& Sonolikar, R. L. (2007). Adaptive neural fuzzy inference system for the detection of inter-turn insulation and bearing wear faults in induction motor. IEEE Transactions on Industrial Electronics, 54(1), $250-258$.

Bayir, R., \& Bay, O. F. (2004). Serial wound starter motor faults diagnosis using artificial neural network. Proceedings of the IEEE International Conference on Mechatronics, 2004. ICM '04., 194-199. doi: 10.1109/ICMECH.2004.1364436

Benbouzid, M. E. (2000). A review of induction motors signature analysis as a medium for faults detection. IEEE TRANSACTIONS ON INDUSTRIAL ELECTRONICS, 47(5), 984-993. doi: 10.1109/41.873206

Betta, G., Liguori, C., Paolillo, A., \& Pietrosanto, A. (2002). A DSP-based FFT-analyzer for the fault diagnosis of rotating machine based on vibration analysis. IEEE Transactions on Instrumentation and Measurement, 51(6), 1316-1322.

Bin, G. F., Gao, J. J., Li, X. J., \& Dhillon, B. S. (2012). Early fault diagnosis of rotating machinery based on wavelet packets-Empirical mode decomposition feature extraction and neural network. Mechanical Systems and Signal Processing, 27, 696-711.

Choudhary, A., Goyal, D., Shimi, S. L., \& Akula, A. (2019). Condition Monitoring and Fault Diagnosis of Induction Motors: A Review. ARCHIVES OF COMPUTATIONAL METHODS IN ENGINEERING, 26(4), 1221-1238. doi: 10.1007/s11831-018-9286-z

Cristalli, C., Paone, N., \& Rodriguez, R. M. (2006). Mechanical fault detection of electric motors by laser vibrometer and accelerometer measurements. MECHANICAL SYSTEMS AND SIGNAL PROCESSING, 20(6), 1350-1361. doi: 10.1016/j.ymssp.2005.11.013

Datta, S., \& Sarkar, S. (2016). A review on different pipeline fault detection methods. Journal of Loss Prevention in the Process Industries, 41, 97-106. doi: https://doi.org/10.1016/j.jlp.2016.03.010

de Jesus Rangel-Magdaleno, J., de Jesus Romero-Troncoso, R., Osornio-Rios, R. A., Cabal-Yepez, E., \& Dominguez-Gonzalez, A. (2010). FPGA-based vibration analyzer for continuous CNC machinery monitoring with fused FFT-DWT signal processing. IEEE Transactions on Instrumentation and Measurement, 59(12), 3184-3194.

Eftekhari, M., Moallem, M., Sadri, S., \& Hsieh, M.-F. (2013). A novel indicator of stator winding inter-turn fault in induction motor using infrared thermal imaging. Infrared Physics \& Technology, 61, 330-336.

Glowacz, A., \& Glowacz, Z. (2017). Diagnosis of stator faults of the single-phase induction motor using acoustic signals. Applied Acoustics, 117, 20-27.

Goyal, D, \& Pabla, B. S. (2016). The vibration monitoring methods and signal processing techniques for structural health monitoring: a review. Archives of Computational Methods in Engineering, 23(4), 585-594.

Goyal, Deepam, Pabla, B. S., Dhami, S. S., \& others. (2017). Condition monitoring parameters for fault diagnosis of fixed axis gearbox: a review. Archives of Computational Methods in Engineering, 24(3), 543-556.

Henao, H., Capolino, G.-A., Fernandez-Cabanas, M., Filippetti, F., Bruzzese, C., Strangas, E., Pusca, R., Estima, J., Riera-Guasp, M., \& Hedayati-Kia, S. (2014). Trends in fault diagnosis for electrical machines: A review of diagnostic techniques. IEEE Industrial Electronics Magazine, 8(2), 31-42.

Hwang, Y.-R., Jen, K.-K., \& Shen, Y.-T. (2009). Application of cepstrum and neural network to bearing fault detection. Journal of Mechanical Science and Technology, 23(10), 2730.

Igba, J., Alemzadeh, K., Durugbo, C., \& Eiriksson, E. T. (2016). Analysing RMS and peak values of vibration signals for condition monitoring of wind turbine gearboxes. Renewable Energy, 91, 90-106. doi: https://doi.org/10.1016/j.renene.2016.01.006

Ilonen, J., Kamarainen, J.-K., Lindh, T., Ahola, J., Kalviainen, H., \& Partanen, J. (2005). Diagnosis tool for motor condition monitoring. IEEE Transactions on Industry Applications, 41(4), 963-971.

Jayakumar, K., \& Thangavel, S. (2017). Industrial drive fault diagnosis through vibration analysis using wavelet transform. Journal of Vibration and Control, 23(12), 2003-2013. doi: 10.1177/1077546315606602

Kochhar, A. K., Atkinson, J., Barrow, G., Burdekin, M., Hannam, R. G., Hinduja, S., Brunn, P., \& Li, L. (1997). Proceedings of the 32nd International MATADOR Conference. Macmillan International Higher Education.

Leung, F. H.-F., Lam, H.-K., Ling, S.-H., \& Tam, P. K.-S. (2003). Tuning of the structure and parameters of a neural network using an improved genetic algorithm. IEEE Transactions on Neural Networks, 14(1), 79-88.

Liu, W. Y., Han, J. G., \& Jiang, J. L. (2013). A novel ball bearing fault diagnosis approach based on auto term window method. Measurement, 46(10), 4032-4037. doi: https://doi.org/10.1016/j.measurement.2013.07.039

Liu, X., Yang, Y., \& Zhang, J. (2018). Resultant vibration signal model based fault diagnosis of a single stage planetary gear train with an incipient tooth crack on the sun gear. RENEWABLE ENERGY, 122, 65-79. doi: 10.1016/j.renene.2018.01.072

Medoued, A., Metatla, A., Boukadoum, A., Bahi, T., \& Hadjadj, I. (2009). Condition monitoring and diagnosis of faults in the electric induction motor. American Journal of Applied Sciences, 6(6), 1133.

Mohammed, O. D., \& Rantatalo, M. (2016). Dynamic response and time-frequency analysis for gear tooth crack detection. Mechanical Systems and Signal Processing, 66-67, 612-624. doi: https://doi.org/10.1016/j.ymssp.2015.05.015

Mousavi, S., Kar, N. C., \& Timusk, M. (2017). A novel parallel modelling-wavelet based mechanical fault detection using stator current signature of induction machine under variable load conditions. J Electr Eng Electr Technol, 6(2), 2-9.

Nejjari, H., \& Benbouzid, M. E. H. (1999). Application of fuzzy logic to induction motors condition monitoring. IEEE Power Engineering Review, 19(6), 52-54.

Palmero, G. I. S., Santamaria, J. J., de la Torre, E. J. M., \& González, J. R. P. (2005). Fault detection and fuzzy rule extraction in AC motors by a neuro-fuzzy ART-based system. Engineering Applications of Artificial Intelligence, 18(7), 867-874.

Pang, J., Li, Y., Jin, X., \& Xu, L. (n.d.). Detection and analysis of typical vibration load of grain harvester based on short-time Fourier method. The International Journal of Electrical Engineering \& Education, 0(0), 0020720919884247. doi: $10.1177 / 0020720919884247$

Prudhom, A., Antonino-Daviu, J., Razik, H., \& Climente-Alarcon, V. (2017). Time-frequency vibration analysis for the detection of motor damages caused by bearing currents. Mechanical Systems and Signal Processing, 84, 747-762. doi: https://doi.org/10.1016/j.ymssp.2015.12.008 
Raghavendra, K., \& Karabasanagouda, B. N. (2014). Frequency response analysis of deep groove ball bearing. Int. J. Sci. Res, 3(8), 1920-1926.

Rao, B. K. N., Pai, P. S., \& Nagabhushana, T. N. (2012). Failure diagnosis and prognosis of rolling-element bearings using Artificial Neural Networks: A critical overview. Journal of Physics: Conference Series, 364(1), 12023.

Sait, A. S., \& Sharaf-Eldeen, Y. I. (2011). A Review of Gearbox Condition Monitoring Based on vibration Analysis Techniques Diagnostics and Prognostics. T. Proulx (Ed.), Rotating Machinery, Structural Health Monitoring, Shock and Vibration, Volume 5 (pp. 307-324). New York, NY: Springer New York.

SaravanaKumar, R., Kumar, K. V., \& Roy, K. (2009). Fuzzy Logic based fault detection in induction machines using Lab view. Int $J$ Comput Sci Netw Secur, 9(9), 226-243.

Saravanan, N., \& Ramachandran, K. I. (2010). Incipient gear box fault diagnosis using discrete wavelet transform (DWT) for feature extraction and classification using artificial neural network (ANN). Expert Systems with Applications, 37(6), 4168-4181.

Saruhan, H., Saridemir, S., Qicek, A., \& Uygur, I. (2014). Vibration Analysis of Rolling Element Bearings Defects. Journal of Applied Research and Technology, 12, 384-395. Retrieved from http://www.scielo.org.mx/scielo.php?script=sci_arttext\&pid=S1665$64232014000300005 \& \mathrm{nrm}=$ iso

Shnibha, R., Albarbar, A., Abouhnik, A., \& Ibrahim, G. (2012). A more reliable method for monitoring the condition of three-phase induction motors based on their vibrations. ISRN Mechanical Engineering, 2012.

Siddiqui, K. M., Sahay, K., \& Giri, V. K. (2015). Rotor broken bar fault detection in induction motor using transformative te chniques. Journal of Electrical Engineering, 15(1), 135-141.

Sudhakar, G. N. D. S., \& Sekhar, A. S. (2009). Coupling Misalignment in Rotating Machines: Modelling, Effects and Monitoring. Noise \& Vibration Worldwide, 40(1), 17-39. doi: 10.1260/0957-4565.40.1.17

Sugumaran, V., \& Ramachandran, K. I. (2011). Effect of number of features on classification of roller bearing faults using SVM and PSVM. Expert Systems with Applications, 38(4), 4088-4096.

Talhaoui, H., Menacer, A., Kessal, A., \& Kechida, R. (2014). Fast Fourier and discrete wavelet transforms applied to sensorless vector control induction motor for rotor bar faults diagnosis. ISA Transactions, 53(5), 1639-1649. doi: https://doi.org/10.1016/j.isatra.2014.06.003

Tiwari, M., Gupta, K., \& Prakash, O. (2000). Effect of radial internal clearance of a ball, bearing on the dynamics of a balanced horizontal rotor. JOURNAL OF SOUND AND VIBRATION, 238(5), 723-756. doi: 10.1006/jsvi.1999.3109

Tsypkin, M. (2011). Induction motor condition monitoring: Vibration analysis technique - A practical implementation. 2011 IEEE International Electric Machines Drives Conference (IEMDC), 406-411. doi: 10.1109/IEMDC.2011.5994629

Unal, M., Onat, M., Demetgul, M., \& Kucuk, H. (2014). Fault diagnosis of rolling bearings using a genetic algorithm optimized neural network. Measurement, 58, 187-196.

Van Hecke, B., Yoon, J., \& He, D. (2016). Low speed bearing fault diagnosis using acoustic emission sensors. Applied Acoustics, 105, $35-44$.

Wang, J., \& Hu, H. (2006). Vibration-based fault diagnosis of pump using fuzzy technique. Measurement, 39(2), 176-185.

Widodo, A., \& Yang, B.-S. (2007). Support vector machine in machine condition monitoring and fault diagnosis. Mechanical Systems and Signal Processing, 21(6), 2560-2574.

Wong, W.-K., Loo, C.-K., Lim, W.-S., \& Tan, P.-N. (2010). Thermal condition monitoring system using log-polar mapping, quaternion correlation and max-product fuzzy neural network classification. Neurocomputing, 74(1-3), 164-177.

Yamamoto, G. K., da Costa, C., \& da Silva Sousa, J. S. (2016). A smart experimental setup for vibration measurement and imbalance fault detection in rotating machinery. Case Studies in Mechanical Systems and Signal Processing, 4, 8-18.

Yang, B.-S., Oh, M.-S., Tan, A. C. C., \& others. (2009). Fault diagnosis of induction motor based on decision trees and adaptive neurofuzzy inference. Expert Systems with Applications, 36(2), 1840-1849.

Zhang, W., Jia, M.-P., Zhu, L., \& Yan, X.-A. (2017). Comprehensive overview on computational intelligence techniques for machinery condition monitoring and fault diagnosis. Chinese Journal of Mechanical Engineering, 30(4), 782-795.

Zhen, D., Guo, J., Xu, Y., Zhang, H., \& Gu, F. (2019). A Novel Fault Detection Method for Rolling Bearings Based on Non-Stationary Vibration Signature Analysis. Sensors, 19(18). doi: 10.3390/s19183994 